\title{
Terahertz spectroscopy from air plasmas created by two-color femtosecond laser
} pulses: The ALTESSE project

Berge, L.; Kaltenecker, Korbinian J.; Engelbrecht, S.; Nguyen, A.; Skupin, S.; Merlat, L.; Fischer, B.; Zhou, Binbin; Thiele, I.; Jepsen, Peter Uhd

\section{Published in:}

EPL

Link to article, DOI:

$10.1209 / 0295-5075 / 126 / 24001$

Publication date:

2019

Document Version

Peer reviewed version

Link back to DTU Orbit

Citation (APA):

Berge, L., Kaltenecker, K. J., Engelbrecht, S., Nguyen, A., Skupin, S., Merlat, L., Fischer, B., Zhou, B., Thiele, I., \& Jepsen, P. U. (2019). Terahertz spectroscopy from air plasmas created by two-color femtosecond laser pulses: The ALTESSE project. EPL, 126(2), [24001]. https://doi.org/10.1209/0295-5075/126/24001

\section{General rights}

Copyright and moral rights for the publications made accessible in the public portal are retained by the authors and/or other copyright owners and it is a condition of accessing publications that users recognise and abide by the legal requirements associated with these rights.

- Users may download and print one copy of any publication from the public portal for the purpose of private study or research.

- You may not further distribute the material or use it for any profit-making activity or commercial gain

- You may freely distribute the URL identifying the publication in the public portal 


\title{
Terahertz spectroscopy from air plasmas created by two-color femtosecond laser pulses: The ALTESSE project
}

\author{
L. Bergé ${ }^{1}$, K. Kaltenecker ${ }^{2}$, S. Engelbrecht ${ }^{3}$, A. NGuyen ${ }^{1}$, S. Skupin $^{4}$, L. Merlat $^{3}$, B. Fischer $^{3}$, B. $^{2}$

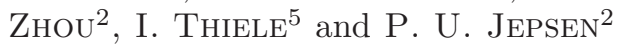 \\ 1 CEA, DAM, DIF - 91297 Arpajon - France \\ 2 DTU Fotonik - Dept. Photonics Engineering, Technical University of Denmark, DK-2800 Kongens Lyngby, Denmark \\ 3 Institut franco-allemand de recherches de Saint-Louis, 5 rue du Général Cassagnou, 68300 Saint-Louis - France \\ 4 Institut Lumière Matière, UMR 5306 Université Lyon 1 - CNRS, Université de Lyon, 69622 Villeurbanne, France \\ 5 Department of Physics, University of Gothenburg, SE-412 96 Göteborg, Sweden
}

PACS $42.65 . \mathrm{Re}$ - Ultrafast processes; optical pulse generation and pulse compression
PACS $32.80 . \mathrm{Fb}$ - Photoionization of atoms and ions
PACS $52.38 .-\mathrm{r}$ - Laser-plasma interactions

\begin{abstract}
Terahertz pulses are very popular because of their numerous applications, for example in security. Located between microwaves and optical waves in the electromagnetic spectrum, their spectral domain can now be exploited for molecular spectroscopy using terahertz emission from plasmas formed by femtosecond laser pulses ionizing gases such as air. Downconversion of broadband optical spectra in a plasma produces intense radiation suitable for the detection of suspect materials remotely. The different physical mechanisms involved to create terahertz radiation by laser-material interaction are reviewed. The new potentialities offered by intense lasers allow the acquisition of unique spectral signatures characterizing various materials.
\end{abstract}

Introduction. - Until the nineties, the domain of terahertz waves (THz), which extends between $100 \mathrm{GHz}$ and a few tens of $\mathrm{THz}$ in the electromagnetic spectrum (fig. 1), was barely explored. This situation radically changed 30 years ago with the advent of the $\mathrm{THz}$ time domain spectroscopy (TDS) and its impact in all sectors of science [1]. Terahertz spectroscopy has indeed become an important tool for studying molecules in the condensed phase. In particular, many chemical, biological, radiological, nuclear and explosive agents that represent warfare hazards exhibit characteristic spectral features in this frequency region, which has thus a strong potential for security applications [2-9]. Various materials such as paper, leather, cotton and synthetic fabrics are transparent in the $\mathrm{THz}$ frequency range, which is one of its main advantages for scanning purposes. $\mathrm{THz}$ photons only excite free carriers, optical phonons, vibrations and molecular rotations. They have a relatively weak energy and the available THz-TDS systems deliver low radiation power $(<1 \mathrm{~mW})$, so that they do not pose a health risk [11]. Besides homeland security, THz-TDS offers a wide range of applications (see fig. 2). For instance, in medical imaging, $\mathrm{THz}$ radiation provides good contrasts for detecting certain cancers [12].

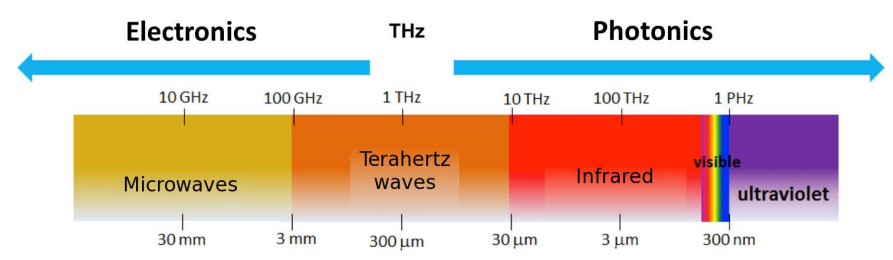

Fig. 1: Terahertz domain in the electromagnetic spectrum.

It can also be used for the detection of greenhouse gases and monitoring of flora [13]. However, because water is opaque to $\mathrm{THz}$ waves, the current challenge in atmospheric $\mathrm{THz}$ technology is to overcome the high absorption of ambient humidity, which can extinguish the $\mathrm{THz}$ radiation over 1-m-long distances. There is thus a growing demand for intense $\mathrm{THz}$ sources. An important requirement in spectroscopy is also to cover large spectral bandwidths, in order to collect as many molecular signatures as possible.

This Perspective article mainly focuses on $\mathrm{THz}$ spectroscopy applications employing laser-based coherent sources and detection techniques. After a review of laserdriven $\mathrm{THz}$ devices and their underlying physics, empha- 
(a)

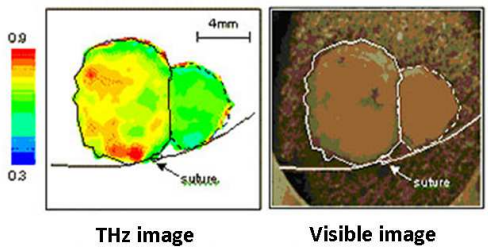

(b)

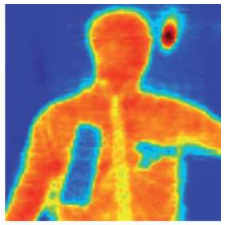

Fig. 2: (a) Terahertz imaging revealing epithelial cancer (red areas), not detected in visible imaging (source: http://www.teraview.com). (b) THz image of an individual concealing suspicious objects under his clothes (Photo Arttu Luukanen, Millilab, Espoo, Finland).

sis is given to recent results obtained in the framework of the French project ALTESSE devoted to the THz spectroscopy of powders, including explosives.

Terahertz transmitters and detectors: from electronics to lasers. - $\mathrm{THz}$ sources can be divided into three categories $[1,14,15]$ : Solid-state electronic devices, quantum-cascade lasers (QCLs) and opticallyinduced THz emitters. Electronic devices, e.g., Schottky diodes $[16,17]$, usually emit weak and narrowband $(<3$ $\mathrm{THz}$ ) radiation. QCLs are unipolar devices where lasing is achieved through intersubband transitions in stacks of semiconductor quantum-well heterostructures $[18,19]$. Although they are frequency-tunable, their emitted pulses, however, remain still narrowband within a few $\mathrm{THz}[20$, 21]. Therefore, only sources of the last category are of significant relevance for THz-TDS.

Optically-based $\mathrm{THz}$ emitters. The first opticallyinduced emitters are photoconductive switches (PCS) that consist of semiconductors equipped with a pair of electrodes between which a high voltage is applied. A femtosecond laser pulse creates electron-hole pairs in the semiconductor, producing a current flow between the electrodes. Since the current is changing typically over one picosecond, the electrodes act as $\mathrm{THz}$-emitting antennas [22]. In general, $\mathrm{THz}$ amplitudes cannot excess the $\mathrm{MV} / \mathrm{m}$ level and the energy yield saturates with the laser pulse energy [23]. THz radiation broadened to 20$30 \mathrm{THz}$ has been demonstrated by using low-temperature grown GaAs or semi-insulating GaAs with ultrashort pulses $[24,25]$. Laser-to-THz conversion efficiency up to $\sim 2 \times 10^{-2}$ can be achieved [26], e.g., by means of plasmonic electrodes, and quasi-single-cycle $\mathrm{THz}$ waveforms with $10 \mu \mathrm{J}$ energy, $33 \mathrm{MV} / \mathrm{m}$ THz field strength have been generated from ZnSe interdigited PCSs [27].

Other $\mathrm{THz}$ transmitters proceed from optical rectification (OR) in nonlinear crystals [28]. Non-centrosymmetric crystals exhibit a $\chi^{(2)}$-nonlinearity, proportional to the square of the exciting laser field. Besides frequency doubling, this nonlinearity leads to down-conversion towards the THz range. Here, two-photon absorption and pump depletion limits the $\mathrm{THz}$ yield [29], together with the phase mismatch between the optical group velocity and the phase velocity of the $\mathrm{THz}$ wave. ZnTe crystals [30] can radiate below $3 \mathrm{THz}$ and offer conversion efficiency around $3 \times 10^{-5}[30,31]$. As an alternative, $\mathrm{LiNbO}_{3}$ crystals have low $\mathrm{THz}$ absorption, higher nonlinear coefficients and higher damage threshold. However, their linear dispersion is strong. Phase-matching conditions can then be optimized by introducing a tilt angle in the pulse front with a diffraction grating $[32,33]$. Typical tilted-pulsefront LiNbO3 THz sources (abbreviated TPF in Table 1 below) provide peak electric fields $>20 \mathrm{MV} / \mathrm{m}$ in the spectral window $\leq 4 \mathrm{THz}$, with high conversion efficiencies $\geq 10^{-2}$ and $>15 \mu \mathrm{J}$ energies $[32,34,35]$. An attractive alternative is the organic crystal DSTMS characterized by nonlinear coefficients one order of magnitude larger than $\mathrm{LiNbO}_{3}$ and good phase-matching. With DSTMS, THz electric fields of $\sim 0.4 \mathrm{GV} / \mathrm{m}$ have been generated [36].

The emitters discussed so far supply a very limited bandwidth of a few THz. Gas-plasma THz emitters overcome this limitation by offering bandwidths exceeding 40 THz. Two decades ago, THz pulses produced at moderate laser intensities $\sim 10^{14} \mathrm{~W} / \mathrm{cm}^{2}$ were created from singlecolor pulses ionizing air under an external strong bias field [37]. A combination of two colors was rapidly proposed, resulting in an increase of the $\mathrm{THz}$ energy by a factor 40 $[38,39]$. Early works on this technique reported peak $\mathrm{THz}$ fields of $15 \mathrm{MV} / \mathrm{m}$, bandwidths as broad as $75 \mathrm{THz}[40,41]$ and $\sim 10^{-4}$ conversion efficiencies. The standard twocolor scheme focuses a fundamental ( $\mathrm{FH})$ laser pulse onto a $\beta$-barium-borate (BBO) crystal placed before the focus of a converging lens. The BBO crystal creates the second harmonic (SH) field and both harmonics co-propagate towards a common focal spot. In air the resulting two-color laser pulse ionizes $\mathrm{O}_{2}$ and $\mathrm{N}_{2}$ molecules at atmospheric pressure close to focus. This creates an electron plasma, called "air plasma", that produces a macroscopic current leading to broadband $\mathrm{THz}$ emission.

This technique has several advantages over conventional THz sources: First, fs-broadband laser pulses generate extended $\mathrm{THz}$ spectra. Second, high amplitude THz fields close to the $\mathrm{GV} / \mathrm{m}$ level can be produced without any risk of damaging the source. Third, the regime of laser filamentation, reached through a local balance between Kerr self-focusing and plasma generation $[42,43]$, makes it possible to generate $\mathrm{THz}$ pulses remotely in the atmosphere [44-46], and thus bypass the absorption of $\mathrm{THz}$ frequencies by water molecules. Fourth, the energy contained in the THz pulse can strongly increase with several laser parameters, including the fundamental pump wavelength [47], the laser pulse duration [48] and the number of colors composing the pump pulse [49].

Last but not least, laser pulses with ultra-high intensities $>10^{18} \mathrm{~W} / \mathrm{cm}^{2}$ can also produce energetic THz waves in relativistic interaction regimes [50]. The advantages of such $\mathrm{THz}$ pulses are manyfold: They are highly energetic, close to the mJ level [51], and broadband with about $70 \%$ of the $\mathrm{THz}$ energy located in the region $<10$ $\mathrm{THz}[52,53]$. Among the main mechanisms generating 
Table 1: Performances of various methods for generating intense $\mathrm{THz}$ waves [58]. THz fields are expressed in $\mathrm{GV} / \mathrm{m}$; frequencies and bandwidth are expressed in $\mathrm{THz} . \mathrm{CE}=$ conversion efficiency; $\mathrm{CF}=$ Central frequency; BW $=$ Bandwidth.

\begin{tabular}{|l|l|l|l|l|}
\hline Method & THz field & CE & CF & BW \\
\hline PCS & $<0.1$ & $10^{-4}$ & $\sim 2$ & $\sim 5$ \\
\hline 2C & 0.8 & $10^{-3}$ & $\sim 5-10$ & $>50$ \\
\hline OR & 8.3 & $10^{-2}$ & $\sim 1-4$ & $\sim 5$ \\
\hline TPF & 0.1 & $10^{-3}$ & $\sim 0.8$ & $\sim 2$ \\
\hline UHI & $>10$ & $5 \times 10^{-4}$ & $1-10$ & $\geq 10$ \\
\hline
\end{tabular}

$\mathrm{THz}$ pulses we find linear mode conversion [52], transient currents at the target rear surface [53] and coherent transition radiation excited when accelerated electrons go across a plasma/vacuum interface with relativistic velocities [54-56]. THz emissions from thin Ti foils was recently measured with $\sim 0.7 \mathrm{~mJ} \mathrm{THz}$ energies, field strengths of $\sim 10 \mathrm{GV} / \mathrm{m}$ and 10-THz bandwidth [57].

Table 1 summarizes the most recent performances (see also [58]) reported from the above technologies.

Detection of intense $\mathrm{THz}$ waveforms and spectra. There are several ways to detect $\mathrm{THz}$ radiation coherently.

The most common technique relies on the ultrafast Pockels effect, where $\mathrm{THz}$ radiation is collected by an electro-optical crystal, for example ZnTe [59,60]. The THz field induces an instantaneous birefringence in the electrooptic medium, which is probed with a second laser pulse split from the pump source. To obtain the $\mathrm{THz}$ electric field in time domain, the signal is recorded as a function of the time delay between the $\mathrm{THz}$ and probe pulse. For measuring broadband, intense $\mathrm{THz}$ pulses, temporal walk-off due to velocity mismatch, however, puts severe conditions on the crystal thickness. This method may also introduce ambiguities linked to phase jumps [61].

Detection by air nonlinearities is possible as well [62-65]. Here, the $\mathrm{THz}$ beam is recombined with the fundamental at a second focus forming the detection region where an electric bias field is applied. This technique is called "ABCD" (Air-Biased Coherent Detection) [66]. A sketch of the ABCD scheme is shown in fig. 5 commented later. A plasma created by a two-color pulse emits $\mathrm{THz}$ radiation towards a sample. This radiation is filtered and directed to the detection part. Here, a probe pulse with controlled delay is focused between two electrodes where an alternating field of $20 \mathrm{kV} / \mathrm{cm}$ is applied. The nonlinear response of air produces second harmonic of the probe pulse that overlaps with the $\mathrm{THz}$ field of interest. The interaction of the fundamental laser field $E_{\omega}$ and the low frequency field components can thus be described by $E_{2 \omega} \propto \chi^{(3)} E_{\omega} E_{\omega} E_{\mathrm{THz}}$, where $\chi^{(3)}$ denotes the third-order susceptibility of air. So, the second harmonic intensity, which is the measured quantity, is proportional to the $\mathrm{THz}$ wave intensity. To get coherent detection, the AC bias voltage is synchronized at half the laser repetition rate.

Another detection technique is the spectral-domain interferometry (SDI), recently extended for $\mathrm{THz}$ field measurements [61,67]. Conventional SDI employs a broadband light source to illuminate a reference surface and a sample, recombined in a Michelson interferometer [14]. In the SDI THz variant, two optical probe pulses with a certain delay are focused into a ZnTe crystal, overlapping with the $\mathrm{THz}$ pulse to be measured. The delay is chosen such that the birefringence induced by the $\mathrm{THz}$ pulse via Pockels effect is experienced by the second pulse only. Then, the two probe pulses are sent into a spectrometer and the $\mathrm{THz}$ pulse is retrieved from interference between the two pulses. Alternatively, $\mathrm{THz}$ waves can also be detected indirectly by $\mathrm{THz}$ Radiation Enhanced Emission of Fluorescence (THz-REEF) that amounts to extracting spectral information on the $\mathrm{THz}$ pulse from the plasma fluorescence [68-70]. Using two color filaments, Liu and co-workers performed a $\mathrm{THz}$ remote sensing at a distance of $10 \mathrm{~m}$ [69]. To end with, let us mention the potential use of solid dielectrics to exploit their strong $\chi^{(3)}$ coefficient. A recent study demonstrated the first solid-state scheme for $\mathrm{THz}$ coherent detection relying on electric-field-induced SH generation by a thin layer of fused silica [71].

Main physical mechanisms. - An ultrashort optical pulse can be converted into the $\mathrm{THz}$ range via specific nonlinearities, namely, (i) the Kerr effect, (ii) photoionization and (iii) plasma waves associated to ponderomotive forces. The relevance of these mechanisms depends on the intensity level engaged, as summarized in fig. 3 .

Four-wave mixing. This mechanism was proposed to interpret the first experiments using two-color laser pulses in air [38]. The polarization vector of the medium contains nonlinear contributions that express in cubic power of the electric field (Kerr effect), namely, $P_{\mathrm{NL}}=\epsilon_{0} \chi^{(3)} E_{L}^{3}$. This nonlinearity mixes the two harmonics, resulting in the production of low frequencies. Ignoring spatial dependencies, a two-color laser field can be modelled as

$$
E_{L}(t)=\sqrt{\frac{2 I_{0}}{c \varepsilon_{0}}} \sum_{j=1,2} a_{j} \mathrm{e}^{-2 \ln 2 \frac{t^{2}}{\tau_{j}^{2}}} \cos \left(j \omega_{0} t+\varphi_{j}\right),
$$

where $I_{0}$ is the pump intensity, $\tau_{j}$ is the FWHM duration of the $j$ th harmonic $(j=1,2), r=a_{2}^{2} / a_{1}^{2}$ is the $\mathrm{SH} / \mathrm{FH}$ intensity ratio with $a_{1}^{2}+a_{2}^{1}=1$, and $\varphi=\varphi_{2}-\varphi_{1}$ is the relative phase between the two carrier waves. In the plane wave approximation $\left(\tau_{j} \rightarrow+\infty\right)$, the Kerr nonlinearity produces a quasi-static component $P_{\mathrm{NL}}^{\mathrm{dc}} \propto a_{1}^{2} a_{2} \cos \varphi$ assimilated to a $\mathrm{THz}$ emitter and maximum for in-phase laser harmonics $(\varphi=0)$. Four-wave mixing is, however, too weak to explain the observed $\mathrm{THz}$ field strengths [39]. Recent experiments [72] showed that its signature is actually present in the $\mathrm{THz}$ spectrum for intensities less than the ionization threshold of air molecules $\sim 5 \times 10^{13}$ $\mathrm{W} / \mathrm{cm}^{2}$. Nevertheless, Kerr contributions, including the effect of delayed Raman scattering, are usually two to 

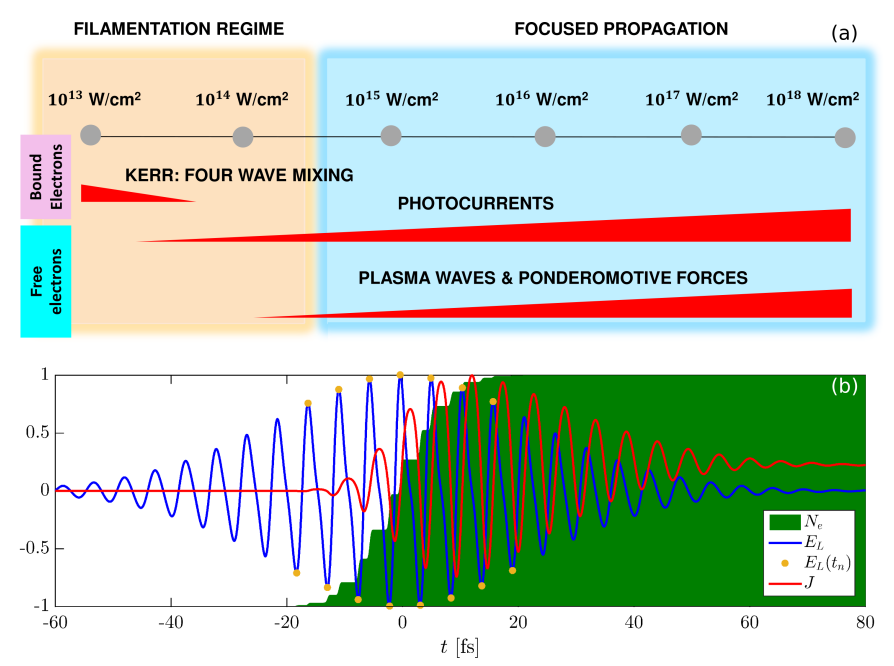

Fig. 3: (a) Mechanisms generating $\mathrm{THz}$ waves by intense twocolor laser pulses, distributed according to the optical intensity. The first region involves the Kerr effect (four-wave mixing) and photoionization. The second region accentuates the contribution of photoionization in the tunnel regime (photocurrents) and involves plasma waves created by ponderomotive forces. (b) Photocurrent process: The two-color electric field generates free electrons in stepwise increase via tunneling ionization occurring near the field extrema at $t=t_{n}$. This builds a slow component of the current that acts as a $\mathrm{THz}$ source.
Given the previous approximation on $N_{e}(t)$, the current $J(t)$ can be divided into two distinct contributions, $J(t) \simeq J_{A}(t)+J_{B}(t)[73,74] . \quad J_{A}(t)$ is the fast current component that mainly describes generation of harmonics due to photoionization. $J_{B}(t)$ depends on the product $\sum_{n} \delta N_{n} v_{f}\left(t_{n}\right)$, where $v_{f}\left(t_{n}\right)$ denotes the electron velocity at instant $t_{n}$, and contains a low frequency component responsible for $\mathrm{THz}$ emission.

Figure 3(b) summarizes $\mathrm{THz}$ generation by a two-color pulse. Ionization appears near the extrema of the laser electric field, inducing peaks in the rate $W(t)$ from which $N_{e}$ increases steplike. Asymmetry in the two-color pulse profile guarantees non-zero velocities $v_{f}\left(t_{n}\right) \propto \sin \varphi$. The low frequency component in $J_{B}(t)$ is maximum for relative phases of $\pi / 2$ between the two colors. This model has been validated by experiments and $3 \mathrm{D}$ simulations [78]. A single-color laser field generates almost no $\mathrm{THz}$ radiation. With two colors, two orders of magnitude can be gained in the $\mathrm{THz}$ energy yield as $\varphi \rightarrow \pi / 2$ [79]. The LC model shows that $\mathrm{THz}$ fields can be enhanced by a judicious arrangement of the laser harmonics. A sawtooth profile can achieve in theory the record conversion rate of $2 \%$ [49]. $\mathrm{THz}$ pulse generation with uncommon frequency ratios of 1:4 and 2:3 has also been experimentally tested [80]. In space, $\mathrm{THz}$ emission takes place along small angles determined by phase-matching conditions and changes in the refraction index due to plasma, depending on the length of the plasma channel [81].

Plasma wakefields. When the laser pump delivers higher intensities $>10^{15} \mathrm{~W} / \mathrm{cm}^{2}$, other nonlinear mechanisms can act as frequency converter. This is the case of plasma waves, for which Eq. (3) must be supplemented by the Lorentz force and quadratic terms in $J$ associated to ponderomotive motions $[82,83]$. Once ionized, the free electrons are dragged by the laser field out of their equilibrium position via the Lorentz force. This displacement of charges forces the electrons to oscillate around ions in the wake of the laser pulse at the electronic plasma frequency $\omega_{\mathrm{pe}}=e^{2} N_{e} / \epsilon_{0} m_{e}$ ( $\epsilon_{0}$ is the vacuum permittivity). This frequency takes typical values between 20 and $60 \mathrm{THz}$ for densities between $10^{17}$ and $10^{18} \mathrm{~cm}^{-3}$. However, it is not guaranteed that, outside the created plasma, such fields can be transmitted to a detector $[84,85]$. This depends on the plasma volume and geometry (e.g., the gradients), the emissivity of which can also be controlled by playing with the transverse laser polarization [87].

\section{Unique spectral signatures. -}

The ALTESSE Project. The identification of energetic materials has become a major issue in dual research, civil and military. A strong axis in this context concerns the acquisition of spectral signatures of explosives or hazardous materials upon large distances. The French project ALTESSE [88] (Air Laser-based TErahertz SpectroScopy of Explosives) aimed at testing the nonlinear terahertz spectroscopy from atmospheric plasmas. Dedicated scientific tasks consisted of (i) optimizing $\mathrm{THz}$ emission in a 


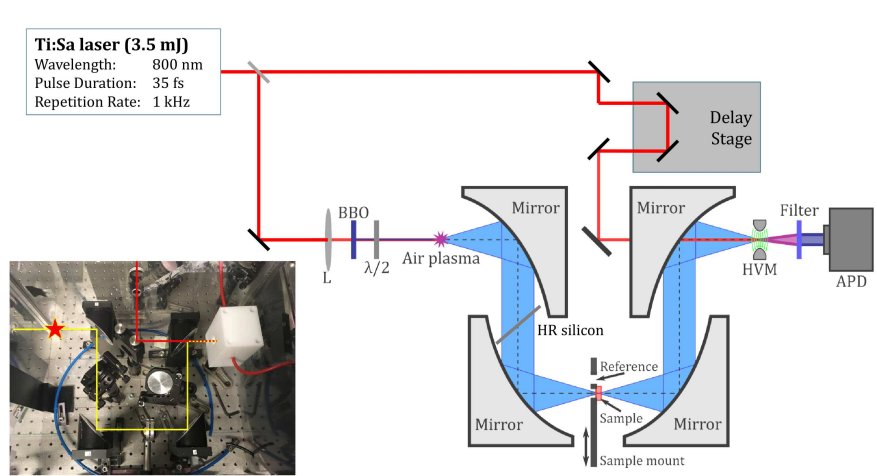

Fig. 4: Experimental device and ABCD detection system in transmission. SH $(400 \mathrm{~nm})$ is produced by a BBO crystal and focused with $\mathrm{FH}(800 \mathrm{~nm})$ in the air by a lens $(\mathrm{L})$. The $\mathrm{THz}$ radiation (blue stripes) from the plasma is filtered by a silicon wafer and collected by a set of off-axis parabolic mirrors. The delay line allows to probe the different instants of the $\mathrm{THz}$ pulse near the detection zone. The coupling between the focused probe beam and a high voltage module (HVM) creates a second harmonic in air detected by an avalanche photodiode (APD).

wide spectral window using two-color pulses; (ii) performing ABCD-based THz-TDS over distances larger than 10 $\mathrm{m}$; (iii) carrying out spectroscopy in transmission and reflection geometries. Many materials have been analyzed in the THz-to-far-infrared domain and we here reveal unique signatures beyond $10 \mathrm{THz}$, using two-color air plasmas.

Broadband THz emitters. The ALTESSE facility operated at DTU/Fotonik [88] involved a generation part (800-nm laser line delivering $35-\mathrm{fs}, 3.5 \mathrm{~mJ}$ pulses at 1 $\mathrm{kHz}$ repetition rate) and a detection part, as shown in fig. 4. After the BBO crystal, a half-wave plate rendered the $\mathrm{FH}$ polarization parallel to the $\mathrm{SH}$. Both colors were focused into air and their relative phase was adjusted by the position of the doubling crystal. THz-TDS was carried out either in transmission or reflection. The samples were prepared in powders mixed with polyethylene, compressed into 1-mm-thick pellets and then mounted on a sample holder. The recorded temporal signals obtained with and without the sample were Fourier transformed to get their characteristic spectra. Figure 5(a) shows a reference $\mathrm{THz}$ field (black curve, without sample) and a $\mathrm{THz}$ field with the sample (red curve), which is thymine (nucleobasis). The reference signal is a single-cycle pulse having a duration of about $200 \mathrm{fs}$. The delay between both signals originates from the optical index of the sample. The spectrum transmitted by the latter shows many dips that correspond to its vibrational modes [see fig. 5(c)]. Here, we display the corresponding absorption coefficient $\alpha(\omega)=-(2 / d) \ln \left[T^{-1}\left|E_{\text {sample }}(\omega) / E_{\text {reference }}(\omega)\right|\right]$, where $d$ and $T$ are the sample thickness and a transmission factor, respectively. The presence of features from $5 \mathrm{THz}$ up to $60 \mathrm{THz}$ demonstrates the rich potential of an ultrabroadband $\mathrm{THz}$ spectroscopy for the precise identification of complex molecules. We also performed $\mathrm{THz}$ spec-
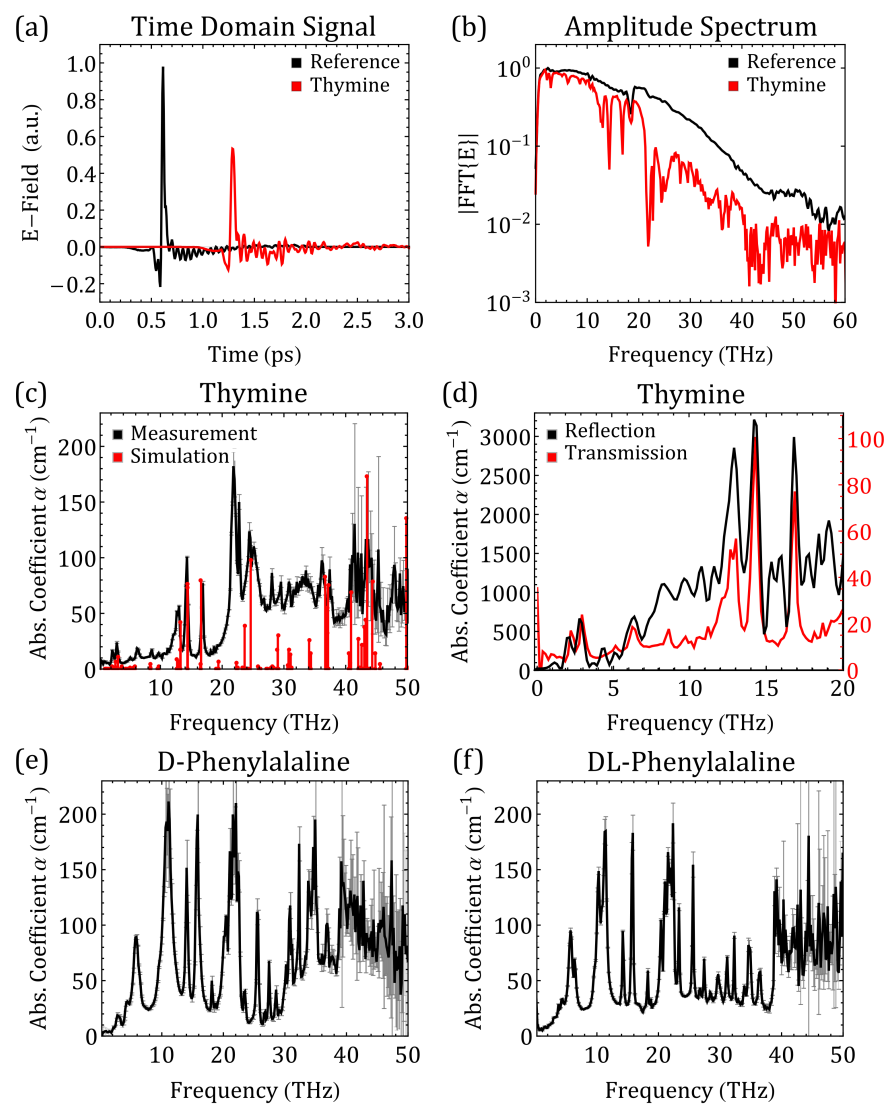

Fig. 5: (a) $\mathrm{THz}$ fields from the reference (nitrogen) and the sample (thymine pellet). (b) Transmission spectra obtained by Fourier transform. (c) Extracted absorption spectrum $[\alpha(\omega)]$. Red bars indicate the position and relative amplitude of CASTEP-calculated phonon modes. (d) Corresponding reflection spectrum. (e,f) show two transmission spectra of stereoisomers: (e) D-phenylalaline and (f) DL-phenylalaline. Thin vertical black lines indicate experimental error bars.

troscopy in reflection geometry by inserting a golden mirror between the last two parabolic mirrors in the setup of fig. 4. The reflected $\mathrm{THz}$ spectrum shown in fig. 5(d) restores thymines's main peaks located within a 20-THzbroad spectral window. Figures $5(\mathrm{e}, \mathrm{f})$ detail the preciseness supplied by this method to discriminate between stereoisomers of the same molecule. Here we observe the four characteristic peaks of the D- and DL-phenylalanine at the same position with similar amplitude. These spectra, however, exhibit differences at low frequencies, which demonstrates the ability of an ABCD-based broadband $\mathrm{THz}$ spectroscopy to distinguish different isomers.

Ab-initio numerical calculations. Computations based on the density functional theory were conducted using the code CASTEP (Cambridge Serial Total Energy Package [89]). CASTEP performs calculations of molecular crystal eigenmodes corresponding to the phonon frequencies (or energies) in a simulated unit cell (60 atoms/cell). These data enable us to compare the calculated phonon modes with the measured absorption spec- 
tra, and also to visualize the molecular motions in the crystal. As an example, figs. 5(c) and 6(a,b) show the absorption spectrum of thymine and of two explosives, PETN and TATB, where their calculated phonon modes are plotted. Both positions and relative amplitudes of the latter are in reasonable agreement with the experimental lines. The four most important modes for thymine are $3.1 \mathrm{THz}$, 13.6 THz, 14.4 THz and 16.6 THz. At $3.1 \mathrm{THz}$, molecules in the unit cell rotate relative to each other, which corresponds to an intermolecular motion of the whole crystal. Going to higher frequencies, the crystal undergoes intramolecular vibrations. PETN owns six distinct peaks in the frequency range of 1 to $16 \mathrm{THz}$, above which the signal becomes noisy (see uncertainty bars). For TATB, the spectrum evidences characteristic signatures at $3.5 \mathrm{THz}$ associated with intermolecular torsion, then at 8.5 and 13 $\mathrm{THz}$ associated with intramolecular angular oscillations.

Remote detection. To end with, figs. 6(c-e) show spectra collected with a terawatt ( $5 \mathrm{~mJ}, 40 \mathrm{fs}$ ) Ti:Sa laser operating at $100 \mathrm{~Hz}$ repetition rate, which was located at 15 meters from the ABCD detection system. The environment was bearing strong variations in temperature and presence of dust and vibrations. We met stability problems in the laser beam energy and directionality triggered by erratic temperature variations of $\pm 3^{\circ} \mathrm{C}$, which led us to acquire spectra over short times $(<10 \mathrm{~min})$ and integration times of $100 \mathrm{~ms}$ (300 ms used at DTU). These spectra were reproduced 3 times for validation. Figure 6(c) displays the first-to-date $18-\mathrm{THz}$ broad $\mathrm{THz}$ spectrum of PETN measured with remote laser source. The 800$\mathrm{nm}$ pump pulse freely propagated a distance $>10 \mathrm{~m}$ before reaching the optical table where the $\mathrm{BBO}$ crystal, the sample and ABCD setup were positioned. There, the twocolor pulse was then focused by a $20-\mathrm{cm}$ lens and created a plasma producing radiation directed to the detection zone. The red curve in fig. 6(c) shows the measured spectrum from a nitrogen-saturated detection chamber. Note the very good agreement with the PETN spectrum measured in clean laboratory conditions (black curve) without longrange pump pulse propagation. Figures $6(\mathrm{~d}, \mathrm{e})$ compare the THz spectra of TATB and ANTA (explosives) measured with the same remote laser source (ISL) and in clean atmosphere (DTU). The remote signals are less resolved due to the above limitations. However, they still contain the main spectral signatures of TATB and even achieve an excellent agreement for the ANTA. Hence, these results demonstrate that, even in noisy atmosphere and over large distances $>10 \mathrm{~m}$, a rapid acquisition of numerous spectral signatures, over a broad bandwidth covering about 20 $\mathrm{THz}$, is feasible and robust.

Conclusion. - Terahertz pulses produced by ultrafast lasers are able to cover a wide range of frequencies, from gigahertz to mid-infrared, and offer rich perspectives for characterizing many materials. In this article, twocolor plasma sources of terahertz radiation driven by photocurrents were discussed. Fully exploiting this nonlin- ear conversion mechanism, the ALTESSE project demonstrated the promising capabilities of portable laser devices for operating an effective, remote detection of suspect materials, including explosives.

$$
* * *
$$

The authors thank ANR and DGA for funding the ASTRID Project "ALTESSE" (ANR-15-ASTR-0009). They also acknowledge support from Laserlab.dk (Danish Center for Laser Infrastructure).

\section{REFERENCES}

[1] Tonouchi M., Nat. Photon., 1 (2007) 9691.

[2] Chen J. et al., Opt. Express, 15 (2007) 12060.

[3] Leahy-Hoppa M. R. et al., Chem. Phys. Lett., 434 (2007) 227.

[4] Frederici J. F. et al., Semicond. Sc. Techn., 20 (2005) S266.

[5] Kemp M. C. et al., Proc. SPIE, 5070 (2003) 44.

[6] Shen Y. C. et al., Appl. Phys. Lett., 86 (2005) 241116.

[7] Fischer B. M. et al., Proc. IEEe, 95 (2007) 1592.

[8] Pereira M. F. and Oleksiy Sh., THz for CBRN and Explosives Detection and Diagnosis, edited by SpRINGer 2017

[9] Liu J. et al., J. Phys.: Conf. Series, 680 (2016) 012030.

[10] Franz M. et al., J. Mol. Struct., 1006 (2011) 34.

[11] BerRy E. et al., J. Laser Appl., 15 (2003) 192.

[12] Yu C. et al., Quant. Imaging Med. Surg., 2 (2012) 33.

[13] Gente R. and Косн M., Plant Methods, 11 (2015) 15.

[14] Hafez H. A. et al., J. Opt., 18 (2016) 093004.

[15] Klymenko $M$ and Shulika Oleksiy $V$. and Sukhoivanov I. A., Terahertz Spectroscopy - A Cutting Edge Technology, edited by InTECH 2017

[16] Crowe Th. et al., Proc. IEEE, 80 (1992) 1827

[17] Maestrini A. et al., Comptes Rendus Physique, 11 (2010) 480

[18] Kazarinov R., Sov. Phys.-Semicond., 5 (1971) 707.

[19] Faist J. et al., Science, 264 (1994) 553.

[20] Walther C. et al., Appl. Phys. Lett., 91 (2007) 131122.

[21] Bachmann D. et al., Optica, 3 (2016) 1087.

[22] Auston D. H. et al., Phys. Rev. Lett., 53 (1984) 1555.

[23] Suen J. Y. et al., Appl. Phys. Lett., 96 (2010) 141103.

[24] Shen Y. C. et al., Appl. Phys. Lett., 83 (2003) 3117.

[25] Hale P. J. et al., Opt. Express, 22 (2014) 26358.

[26] Yardimci N. T. et al., IEEE Trans. Terahertz Sci. Technol., 5 (2015) 223.

[27] Ropagnol X et al., Opt. Express, 24 (2016) 11299.

[28] Boyd R. W., Nonlinear Optics, edited by ACAdemic Press (San Diego) 2008

[29] Sun F. G. et al., CLEO 2000 Tech. Digest, 39 (2000) 479.

[30] Blanchard F et al., Opt. Express, 15 (2007) 13212.

[31] Loeffler T. et al., Opt. Express, 13 (2005) 5353.

[32] Hebling J. et al., Appl. Phys. B, 78 (2004) 593.

[33] Hebling J. et al., Opt. Express, 10 (2002) 1161.

[34] Blanchard F. et al., Opt. Lett., 39 (2014) 4333.

[35] Huang S. W. et al., Opt. Lett., 38 (2013) 796.

[36] Vicario C. et al., Phys. Rev. Lett., 112 (2014) 213901.

[37] LÖffler T. and Jacob F. and Roskos H. G., Appl. Phys. Lett., 77 (2000) 453. 

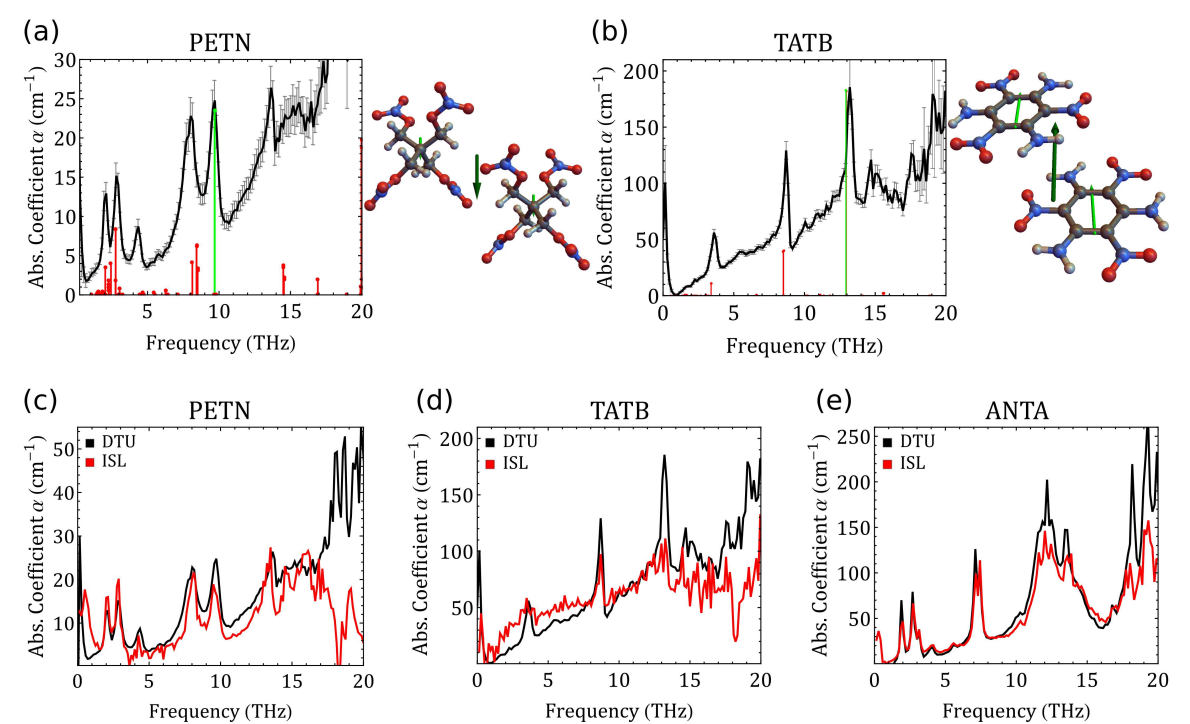

Fig. 6: Absorption spectra (black curves) and simulated phonon modes (red bars) for (a) PETN and (b) TATB. Thin vertical black lines correspond to experimental error bars. On the right, characteristic molecular structures illustrating dipole excitations are shown (red: O, blue: N, grey: $\mathrm{H}$ atoms). Arrows indicate the center of mass dipole moments: the big arrow at center shows the total dipole moment of the unit cell related to the green absorption line; the two smaller arrows in the center of each molecule are the respective net dipole moments. (c-e) Absorption spectra collected in transmission far from the laser output for (c) PETN, (d) TATB and (e) ANTA. Red curves are spectra measured at Institut Saint-Louis (ISL - France) with the Ti:Sa source located at a distance of $15 \mathrm{~m}$ from the ABCD system. Black curves are spectra measured in the laboratory over optical propagation paths of less than $1 \mathrm{~m}$ in clean atmosphere (DTU/Fotonik - Denmark).

[38] Cook D. J. and Hochstrasser R. M., Opt. Lett., 25 (2000) 1210.

[39] Kress M. et al., Opt. Lett., 29 (2004) 1120.

[40] KIM K. Y. et al., Nat. Photon., 2 (2008) 605.

[41] Kiм K. Y. et al., Opt. Express, 15 (2007) 4577.

[42] Braun A et al., Opt. Lett., 20 (1995) 73

[43] Bergé L. et al., Rep. Prog. Phys., 70 (2007) 1633.

[44] Bergé L. et al., Phys. Rev. Lett., 110 (2013) 073901.

[45] D'Amico C. et al., Phys. Rev. Lett., 98 (2007) 235002.

[46] Daigle J.-F. et al., Opt. Express, 20 (2012) 6825.

[47] Clerici M. et al., Phys. Rev. Lett., 110 (2013) 253901.

[48] Wang T.-J. et al., Appl. Phys. Lett., 97 (2010) 111108.

[49] González de Alaiza Martínez P. et al., Phys. Rev. Lett., 114 (2015) 183901.

[50] Hamster H. et al., Phys. Rev. Lett., 71 (1993) 2725.

[51] Gopal A. et al., Opt. Lett., 38 (2013) 4705.

[52] Liao G. Q. et al., Phys. Rev. Lett., 114 (2015) 255001.

[53] Gopal A. et al., Phys. Rev. Lett., 111 (2013) 074802.

[54] Leemans W. P. et al., Phys. Rev. Lett., 91 (2003) 074802.

[55] Liao G. Q. et al., Phys. Rev. Lett., 116 (2016) 205003.

[56] Déchard J. et al., Phys. Rev. Lett., 120 (2018) 144801.

[57] Herzer S. et al., New J. Phys., 20 (2018) 063019.

[58] Zhang X. C. et al., Nat. Photon., 11 (16) 2017.

[59] Wu Q. and Zhang X.-C., Appl. Phys. Lett., 70 (1997) 1784.

[60] Leitenstorfer A. et al., Appl. Phys. Lett., 74 (1999) 1516.

[61] Sharma G. et al., Opt. Lett., 37 (2012) 4338.

[62] XIE X. et al., Phys. Rev. Lett., 96 (2006) 075005.

[63] Dai J.-M. et al., Phys. Rev. Lett., 97 (2006) 103903.

[64] Karpowicz N. et al., Appl. Phys. Lett., 92 (2008) 011131.

[65] LiU K. and Zhang X.-C., THz for CBRN and Explosives
Detection and Diagnosis, edited by SPRINGER (Dordrecht, Netherlands) 2017

[66] Klarskov P. et al., New J. Phys., 15 (2013) 075012.

[67] Sharma G. et al., Opt Lett., 38 (2013) 2705.

[68] Liu J. and Zhang X.-C., Phys. Rev. Lett, 103 (2009) 235002.

[69] Liu J. et al., Nat. Photon., 4 (2010) 627.

[70] DAI J. et al., IEEE J. Sel. Top. Quantum, 17 (2011) 183.

[71] Tomasino A. et al., Optica, 4 (2017) 1358.

[72] Andreeva V. A. et al., Phys. Rev. Lett., 116 (2016) 063902.

[73] Babushkin I. et al., New J. Phys., 13 (2011) 123029.

[74] Nguyen A. et al., Opt. Express, 25 (2017) 4720.

[75] Keldysh L. V., Sov. Phys. JETP, 20 (1965) 1307.

[76] Perelomov A. M. et al., Sov. Phys. JeTP, 23 (1966) 924.

[77] Thomson M. D. et al., Laser Photon. Rev., 1 (2007) 349.

[78] Babushinin I. et al., Phys. Rev. Lett., 105 (2010) 053903.

[79] Debayle A. et al., Opt. Express, 22 (2014) 13691.

[80] Zhang L.-L. et al., Phys. Rev. Lett., 119 (2017) 235001.

[81] You Y. S., Phys. Rev. Lett, 109 (2012) 183902.

[82] D'Amico C. et al., New J. Phys., 10 (2007) 013015.

[83] Sprangle P. et al., Phys. Rev. E, 69 (2004) 066415.

[84] Thiele I. et al., Phys. Rev. E, 94 (2016) 063202.

[85] Miao Ch. et al., Phys. Plasmas, 20 (2018) 063019.

[86] González de Alaiza Martínez P. et al., Scient. Reports, 6 (2016) 26743.

[87] Thiele I. et al., Optica, 5 (2018) 1617.

[88] ALTESSE Website: Www.AGENCEnAtionaleRECHERChE.FR/PROJECT-ANR-15-ASTR-0009,.

[89] Clark S. J. et al., Zeit. Kristal., 220 (2005) 567. 